\title{
Approximate Solution of Perturbed Volterra-Fredholm Integrodifferential Equations by Chebyshev-Galerkin Method
}

\author{
K. Issa ${ }^{1}$ and F. Salehi ${ }^{2}$ \\ ${ }^{1}$ Department of Statistics and Mathematical Sciences, Kwara State University, PMB 1530, Malete, Ilorin, Kwara State, Nigeria \\ ${ }^{2}$ Department of Mathematics, Islamic Azad University, Darab Branch, Darab, Iran
}

Correspondence should be addressed to K. Issa; issakazeem@yahoo.com

Received 4 October 2016; Accepted 22 December 2016; Published 12 January 2017

Academic Editor: Liwei Zhang

Copyright (C) 2017 K. Issa and F. Salehi. This is an open access article distributed under the Creative Commons Attribution License, which permits unrestricted use, distribution, and reproduction in any medium, provided the original work is properly cited.

In this work, we obtain the approximate solution for the integrodifferential equations by adding perturbation terms to the right hand side of integrodifferential equation and then solve the resulting equation using Chebyshev-Galerkin method. Details of the method are presented and some numerical results along with absolute errors are given to clarify the method. Where necessary, we made comparison with the results obtained previously in the literature. The results obtained reveal the accuracy of the method presented in this study.

\section{Introduction}

Integrodifferential equations (IDEs) arise in many branches of mathematical sciences such as financial mathematics, mathematical modeling, and control theory, which are usually difficult to solve analytically, so numerical approach is required. Different methods have been used to obtain the solution of both linear and nonlinear IDEs such as Galerkin method [1-4], homotopy perturbation [5, 6], Tau method [79], spline collocation method [10-12], Taylor collocation [1317], finite element approach [18-23], Legendre polynomials [24], variational iteration method [25], Haar wavelets [26], Krein's method [27], and Bessel collocation method [28], to mention a few.

The present work is motivated by the desire to obtain numerical solutions to initial value problems for integrodifferential equations via perturbed Chebyshev-Galerkin method. This paper is organized as follows. In Section 2, Chebyshev polynomial is discussed. In Section 3, preliminary steps towards application of the perturbed ChebyshevGalerkin method are introduced. In Section 4, some numerical results are provided to demonstrate the efficiency and accuracy of using perturbed Chebyshev-Galerkin method and compared with those of $[1,29]$ and lastly, Section 5 is the conclusion.

\section{Chebyshev Polynomial}

Chebyshev polynomials are widely used in applications in mathematics, mathematical physics, engineering, and computer science. Chebyshev polynomial is an orthogonal polynomial which satisfy the recurrence relation

$$
\begin{gathered}
T_{n}(x)=\cos \left[n \cos ^{-1}\left(\frac{2 x-(a+b)}{b-a}\right)\right], \quad a \leq x \leq b, \\
T_{n+1}(x)=\left(\frac{2 x-(a+b)}{b-a}\right) T_{n}(x)-T_{n-1}(x), \quad n \geq 1 .
\end{gathered}
$$

In recent years, a lot of attention has been devoted to the study of Chebyshev methods to investigate various scientific models. Using these methods made it possible to solve differential equations of different forms $[1,7,30-32]$, irrespective of the order of the differential equations.

In certain applications we need expressions for the products like $T_{i}(x) T_{j}(x)$, which comes easily from

$$
T_{i}(x) T_{j}(x)=\frac{1}{2}\left[T_{i+j}(x)+T_{i-j}(x)\right] .
$$




\section{Perturbed Chebyshev-Galerkin Method}

We will consider the numerical solution of a class of linear Volterra-Fredholm integrodifferential initial value problems of the form

$$
\begin{aligned}
\sum_{l=0}^{m} p_{l}(x) \frac{d^{l}}{d x^{l}} \phi(x)= & f(x)+\lambda_{1} \int_{a}^{b} \tau_{1}(x, t) \phi(t) d t \\
& +\lambda_{2} \int_{a}^{x} \tau_{2}(x, t) \phi(t) d t, \\
\phi^{j}(a)= & \gamma_{j}, \quad j=0,1, \ldots, m-1,
\end{aligned}
$$

where $\phi(x)$ is the unknown function, $p_{l}(x), f(x)$, and $\tau_{k}(x, t)$, $k=1,2$, are known functions, $m$ is the order of (3), and $\lambda_{k}, k=1,2$, are real numbers. Unless otherwise stated, $x$ will always be the independent variable of the functions which appear throughout this paper and will be defined in a finite interval $[a, b]$. Moreover suppose that $\phi_{n}(x)$ be the approximate solution of degree $n$ to $\phi(x)$, so we write

$$
\phi_{n}(x)=\sum_{i=0}^{n} \beta_{i} T_{i}\left(\frac{2 x-(a+b)}{b-a}\right) \text {, }
$$

where $\beta_{i}$ are determined by adding perturbation terms

$$
H_{n}(x)=\sum_{l=0}^{m} \mu_{l+1} T_{n-m+l+1}\left(\frac{2 x-(a+b)}{b-a}\right)
$$

to the right hand side of (3); we obtain

$$
\begin{aligned}
\sum_{l=0}^{m} p_{l}(x) \frac{d^{l}}{d x^{l}} \phi_{n}(x)= & f(x)+\lambda_{1} \int_{a}^{b} \tau_{1}(x, t) \phi_{n}(t) d t \\
& +\lambda_{2} \int_{a}^{x} \tau_{2}(x, t) \phi_{n}(t) d t \\
& +H_{n}(x) .
\end{aligned}
$$

Applying Chebyshev-Galerkin approach discussed in [1], that is, multiplying both sides of $(7)$ by $T_{s}((2 x-(a+b)) /(b-a))$, $s=m, m+1, \ldots, n+m+1$, and then integrating the resulting equation over interval $[a, b]$, we obtain

$$
\begin{aligned}
\int_{a}^{b} & {\left[\sum_{l=0}^{m} p_{l}(x) \sum_{i=0}^{n}\left(\frac{2}{b-a}\right)^{l} \beta_{i} \frac{d^{l}}{d x^{l}} T_{i}\left(\frac{2 x-(a+b)}{b-a}\right)\right.} \\
& -\lambda_{1} \int_{a}^{b} \tau_{1}(x, t) \sum_{i=0}^{n} \beta_{i} T_{i}\left(\frac{2 t-(a+b)}{b-a}\right) d t \\
& -\lambda_{2} \int_{a}^{x} \tau_{2}(x, t) \sum_{i=0}^{n} \beta_{i} T_{i}\left(\frac{2 t-(a+b)}{b-a}\right) d t \\
& \left.-\sum_{l=0}^{m} \mu_{l+1} T_{n-m+l+1}\left(\frac{2 x-(a+b)}{b-a}\right)\right]
\end{aligned}
$$

TABLE 1: Absolute errors for Example 1 at different $n$.

\begin{tabular}{lccc}
\hline$x$ & $n=4$ & $n=8$ & $n=12$ \\
\hline 0 & 0 & 0 & 0 \\
0.1 & $7.8696 e-06$ & $2.9221 e-08$ & $3.9291 e-11$ \\
0.2 & $1.3565 e-04$ & $7.2475 e-08$ & $8.0337 e-12$ \\
0.3 & $1.9086 e-04$ & $2.5687 e-08$ & $7.0680 e-11$ \\
0.4 & $1.4192 e-04$ & $2.7660 e-08$ & $1.8044 e-11$ \\
0.5 & $3.6209 e-05$ & $5.6989 e-08$ & $6.6114 e-11$ \\
0.6 & $5.5319 e-05$ & $1.2399 e-07$ & $9.7743 e-11$ \\
0.7 & $7.6735 e-05$ & $7.0373 e-08$ & $6.7821 e-11$ \\
0.8 & $1.4665 e-05$ & $6.0573 e-08$ & $1.4743 e-10$ \\
0.9 & $8.1616 e-05$ & $1.5553 e-07$ & $1.2997 e-10$ \\
1.0 & $8.5267 e-05$ & $1.5711 e-07$ & $1.8751 e-10$ \\
\hline
\end{tabular}

$$
\begin{aligned}
& \cdot T_{s}\left(\frac{2 x-(a+b)}{b-a}\right) d x=f(x) \\
& \cdot T_{s}\left(\frac{2 x-(a+b)}{b-a}\right) d x,
\end{aligned}
$$

$$
s=m, m+1, \ldots, n+m+1 .
$$

From (8), we have

$$
\theta \beta=F,
$$

where $\theta$ is a matrix of $(n+2) \times(n+m+2), \beta$ and $F$ are column matrices of $(n+2) \times 1$, and the other equations are derived from the initial conditions (4); that is,

$$
\begin{aligned}
& \frac{d^{j}}{d x^{j}} \phi_{n}(a) \\
& =\left.\sum_{i=0}^{n}\left(\frac{2}{b-a}\right)^{j} \beta_{i} \frac{d^{j}}{d x^{j}} T_{i}\left(\frac{2 x-(a+b)}{b-a}\right)\right|_{x=a}=\gamma_{j}, \\
& j=0,1, m-1 .
\end{aligned}
$$

Substitute the values of $\beta_{0}, \beta_{1}, \ldots, \beta_{n}$ obtained from (9) and (10) in (5) to obtain the approximate solution of degree $n$.

\section{Numerical Examples}

Example 1 (see $[1,33]$ ). Consider the Fredholm integrodifferential equation

$$
\begin{aligned}
\phi^{\prime}(x)= & \phi(x)-\frac{1}{2} x+\frac{1}{1+x}-\log (1+x) \\
& +\frac{1}{(\log (2))^{2}} \int_{0}^{1} \frac{x}{1+t} \phi(t) d t, \quad 0 \leq x \leq 1
\end{aligned}
$$

subject to initial condition

$$
\begin{aligned}
& \phi(0)=0 \text { and whose exact solution is } \\
& \phi(x)=\log (1+x) .
\end{aligned}
$$

The absolute errors are tabulated in Table 1 at different $n$. Table 5 exhibits a comparison between the errors obtained by 
TABLE 2: Absolute errors for Example 2 at different $n$.

\begin{tabular}{lccc}
\hline$x$ & $n=4$ & $n=8$ & $n=12$ \\
\hline 0 & 0 & 0 & 0 \\
0.1 & $2.2012 e-05$ & $2.3599 e-10$ & $5.9771 e-17$ \\
0.2 & $1.5422 e-04$ & $2.2695 e-10$ & $1.0337 e-16$ \\
0.3 & $2.2010 e-04$ & $2.8800 e-11$ & $9.2897 e-17$ \\
0.4 & $1.7484 e-04$ & $4.1892 e-10$ & $9.1015 e-17$ \\
0.5 & $5.7582 e-05$ & $5.7543 e-11$ & $1.2640 e-17$ \\
0.6 & $5.4134 e-05$ & $2.8830 e-10$ & $6.0745 e-17$ \\
0.7 & $8.7363 e-05$ & $1.1093 e-10$ & $1.2000 e-16$ \\
0.8 & $1.3365 e-05$ & $3.5180 e-10$ & $7.3259 e-17$ \\
0.9 & $1.1663 e-04$ & $9.5773 e-11$ & $8.8650 e-17$ \\
1.0 & $1.4003 e-04$ & $1.4225 e-10$ & $3.1275 e-17$ \\
\hline
\end{tabular}

using the perturbed Chebyshev-Galerkin and using Galerkin method [1]. Figure 1 shows the absolute errors at different $n$ and Figure 1(b) presents the perturbed Chebyshev-Galerkin method and exact solutions.

Example 2 (see $[1,34])$. Consider the Volterra integrodifferential equation

$$
\begin{aligned}
\phi^{\prime}(x)-\int_{0}^{x} \phi(t) d t=1-2 x \sin (x), & \\
& 0 \leq x \leq 1, \phi(0)=0,
\end{aligned}
$$

whose exact solution is $\phi(x)=x \cos (x)$.

The numerical results for the absolute errors are displayed in Table 2 for different values of $n$; comparison of maximum absolute errors is tabulated in Table 5 while Figure 2 exhibits the perturbed Chebyshev-Galerkin and exact solutions and the maximum absolute errors at different $n$.

Example 3 (see $[1,34]$ ). Consider the Volterra integrodifferential equation

$$
\begin{aligned}
\phi^{\prime}(x)+\int_{0}^{x} t \phi(t) d t=-1+\frac{1}{2} x^{2}-x \exp (x), & \\
0 & \leq x \leq 1, \phi(0)=0,
\end{aligned}
$$

whose exact solution is $\phi(x)=1-\exp (x)$.

The computational results for the absolute errors are summarized in Table 3 for different values of $n$ and comparison of maximum absolute errors are tabulated in Table 5 while Figure 3 exhibits the perturbed Chebyshev-Galerkin and exact solutions and the maximum absolute error at different $n$.

Example 4 (see $[13,29])$. Consider Fredholm-Volterra integrodifferential equation

$$
\begin{aligned}
\phi^{\prime \prime}(x)+x \phi^{\prime}(x)- & x \phi(x) \\
=e^{x}-2 \sin (x) & +\int_{-1}^{1} \sin (x) e^{t} \phi(t) d t, \\
& -1 \leq x \leq 1, \phi(0)=1, \phi^{\prime}(0)=1 .
\end{aligned}
$$

The exact solution is $\phi(x)=e^{x}$.
TABLE 3: Absolute errors for Example 3 at different $n$.

\begin{tabular}{lccc}
\hline$x$ & $n=4$ & $n=8$ & $n=12$ \\
\hline 0 & 0 & 0 & 0 \\
0.1 & $5.1479 e-06$ & $4.8686 e-11$ & $8.9783 e-18$ \\
0.2 & $5.4286 e-05$ & $5.0102 e-11$ & $1.4736 e-17$ \\
0.3 & $8.1338 e-05$ & $1.4487 e-13$ & $1.4206 e-17$ \\
0.4 & $6.5339 e-05$ & $8.6061 e-11$ & $1.2362 e-17$ \\
0.5 & $1.7563 e-05$ & $9.9855 e-12$ & $1.4139 e-18$ \\
0.6 & $3.2690 e-05$ & $7.0793 e-11$ & $1.0652 e-17$ \\
0.7 & $5.3816 e-05$ & $1.2859 e-11$ & $1.6736 e-17$ \\
0.8 & $2.9273 e-05$ & $6.8704 e-11$ & $1.2782 e-17$ \\
0.9 & $2.3223 e-05$ & $3.3909 e-11$ & $1.1424 e-17$ \\
1.0 & $3.0485 e-05$ & $1.5662 e-11$ & $2.1410 e-18$ \\
\hline
\end{tabular}

TABle 4: Absolute errors for Example 4 at different $n$.

\begin{tabular}{lccc}
\hline$x$ & $n=4$ & $n=8$ & $n=12$ \\
\hline 0 & 0 & 0 & 0 \\
0.1 & $2.4216 e-05$ & $5.5536 e-09$ & $1.1520 e-12$ \\
0.2 & $5.6674 e-05$ & $4.4620 e-08$ & $9.1630 e-12$ \\
0.3 & $7.5702 e-05$ & $1.4986 e-07$ & $3.0638 e-11$ \\
0.4 & $1.0165 e-04$ & $3.5091 e-07$ & $7.1708 e-11$ \\
0.5 & $1.8402 e-04$ & $6.7437 e-07$ & $1.3786 e-10$ \\
0.6 & $3.8724 e-04$ & $1.1437 e-06$ & $2.3382 e-10$ \\
0.7 & $7.7496 e-04$ & $1.7782 e-06$ & $3.6348 e-10$ \\
0.8 & $1.3927 e-03$ & $2.5927 e-06$ & $5.2990 e-10$ \\
0.9 & $2.2486 e-03$ & $3.5978 e-06$ & $7.3533 e-10$ \\
1.0 & $3.2922 e-03$ & $4.8012 e-06$ & $9.8128 e-10$ \\
\hline
\end{tabular}

For numerical results see Tables 4 and 6 while Figure 4 displays the approximant and the maximum errors as compared with the results from the literature [29].

\section{Conclusion}

This paper has discussed how the perturbed ChebyshevGalerkin method can be applied for obtaining solutions of integral and integrodifferential equations. The formulation and implementation of the scheme are illustrated. The proposed method was tested using some problems with results. This paper discussed how the integrodifferential equation with variable and constant coefficients can be solved using the perturbed Chebyshev-Galerkin method. Maple and Matlab had been used to obtain the approximate solution and for plotting the graphs, respectively. Numerical results demonstrate that our method is an accurate and reliable numerical technique for solving $m$ th order integrodifferential and integral equations. Finally, because of the accuracy and simplicity of the elegant method presented in this study, we recommend its application in finding the approximate solution to integrodifferential and integral equations. 


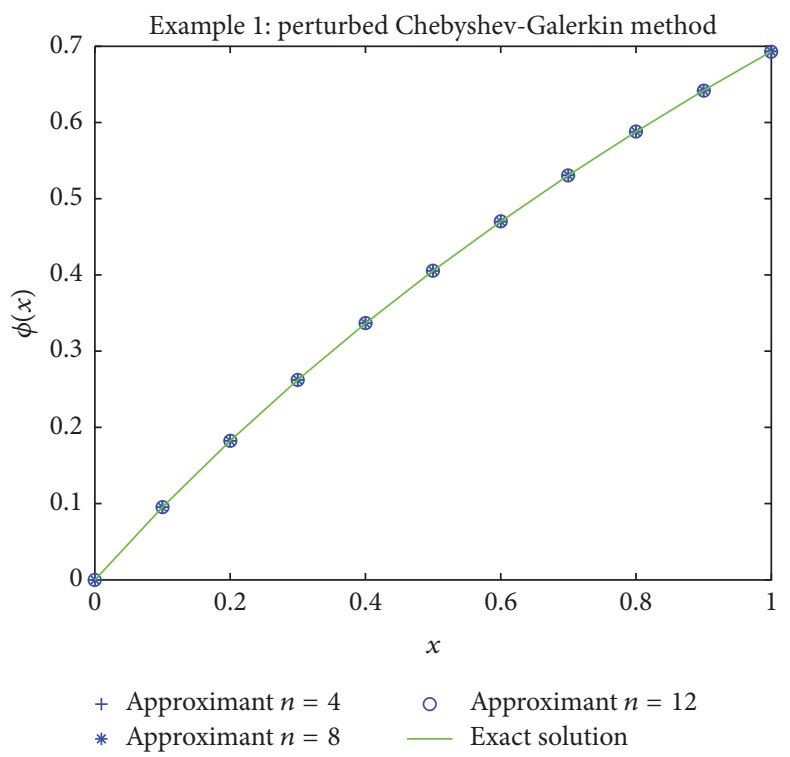

(a)

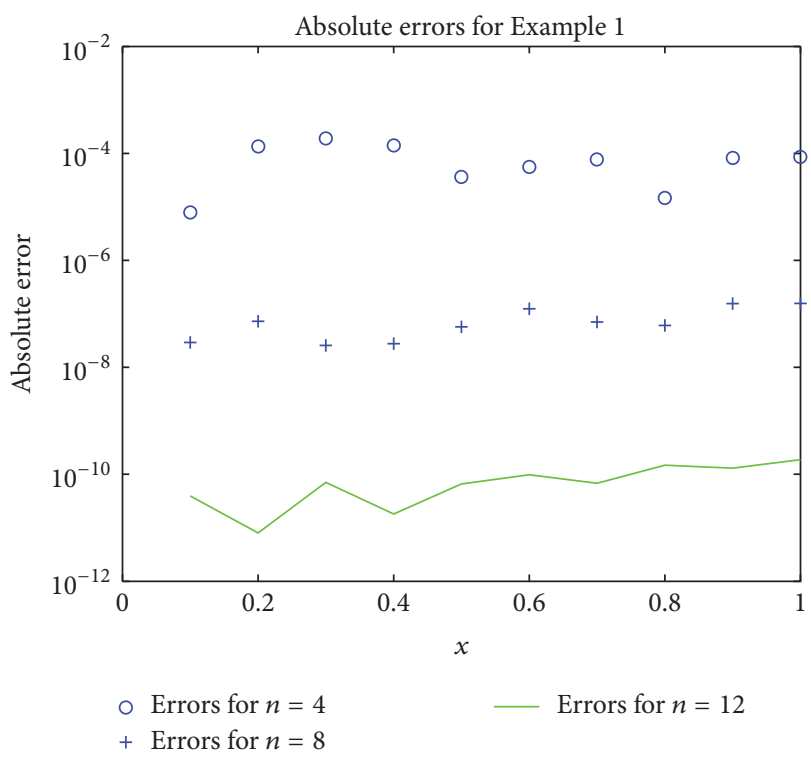

(b)

FIGURE 1: The approximate solution for Example 1 and its absolute errors for different $n$.

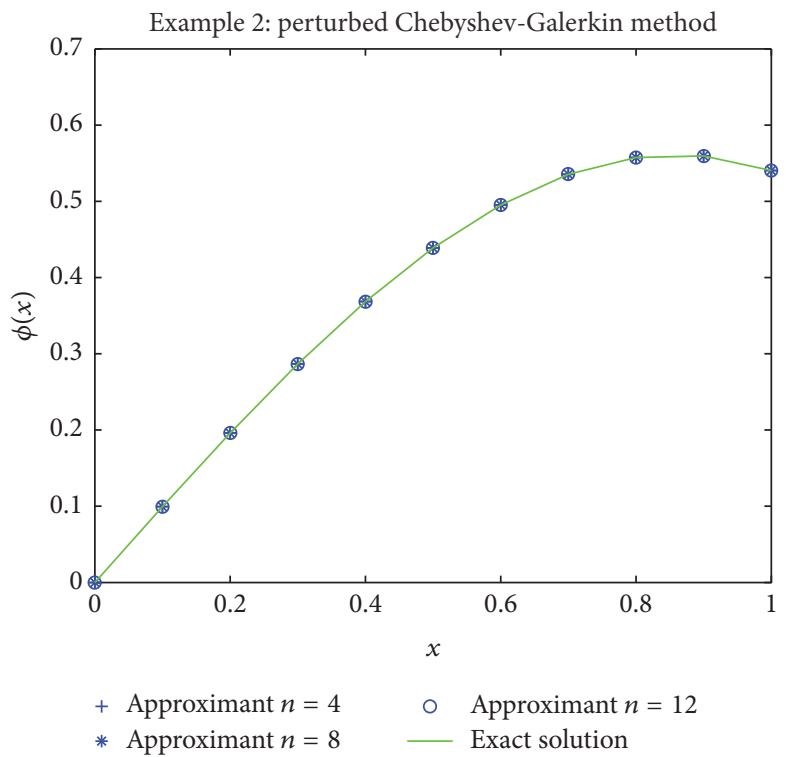

(a)

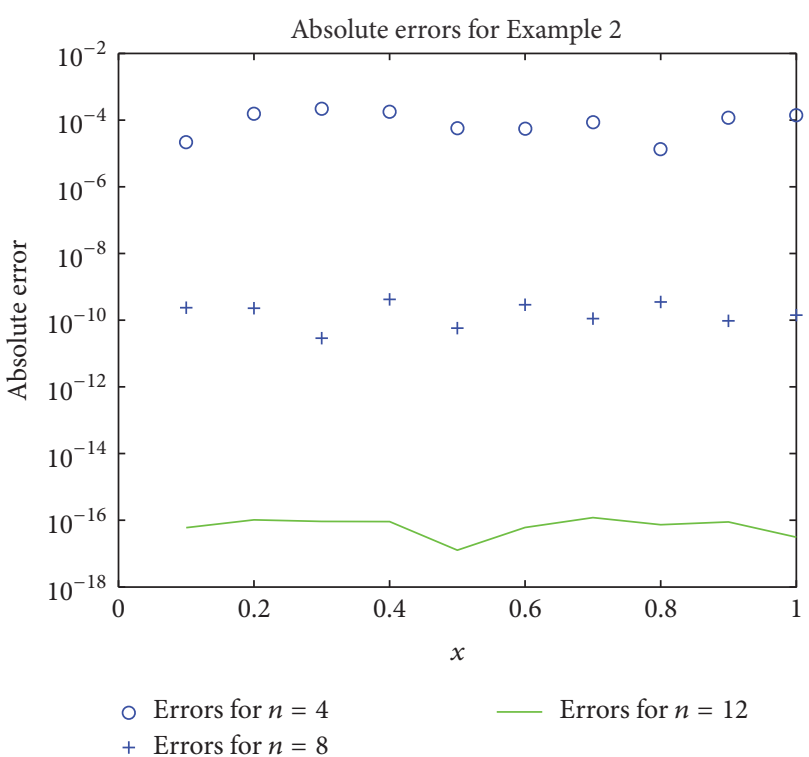

(b)

Figure 2: The approximate solution for Example 2 and its absolute errors for different $n$.

TABle 5: Comparison of absolute maximum errors for Examples 1-3 at different $n$.

\begin{tabular}{lcccccc}
\hline & \multicolumn{2}{c}{ Example 1 } & \multicolumn{2}{c}{ Example 2 } & \multicolumn{2}{c}{ Example 3 } \\
& Present method & Galerkin method [1] & Present method & Galerkin method [1] & Present method & Galerkin method [1] \\
\hline 4 & $1.9086 e-04$ & $4.629 e-3$ & $2.2010 e-04$ & $2.300 e-3$ & $8.1338 e-05$ & $7.204 e-4$ \\
8 & $1.5711 e-07$ & $5.611 e-6$ & $4.1892 e-10$ & $1.382 e-8$ & $8.6061 e-11$ & $2.311 e-9$ \\
12 & $1.8751 e-10$ & $5.930 e-09$ & $1.2000 e-16$ & $8.087 e-15$ & $1.6736 e-17$ & $9.235 e-16$ \\
\hline
\end{tabular}




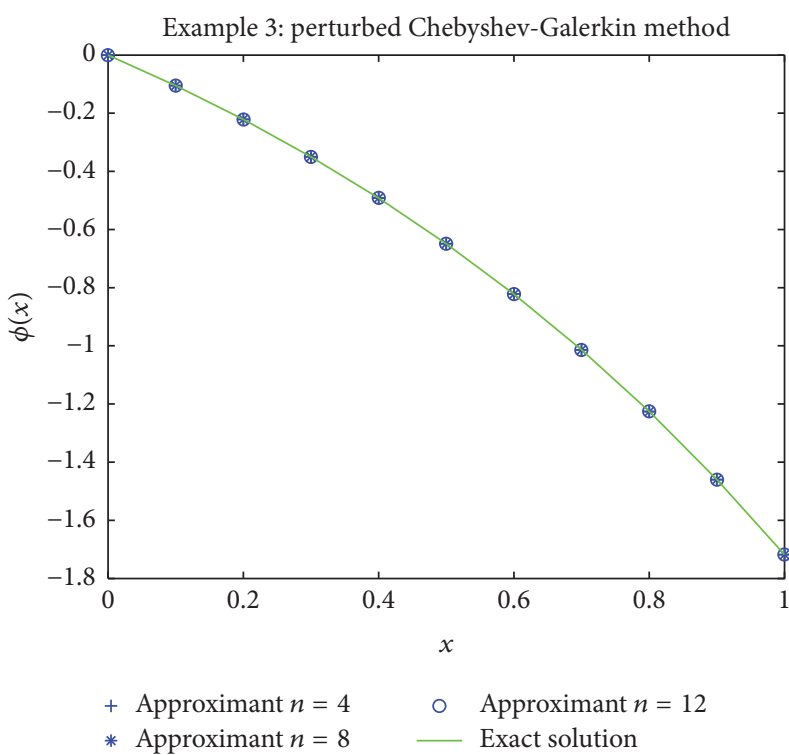

(a)

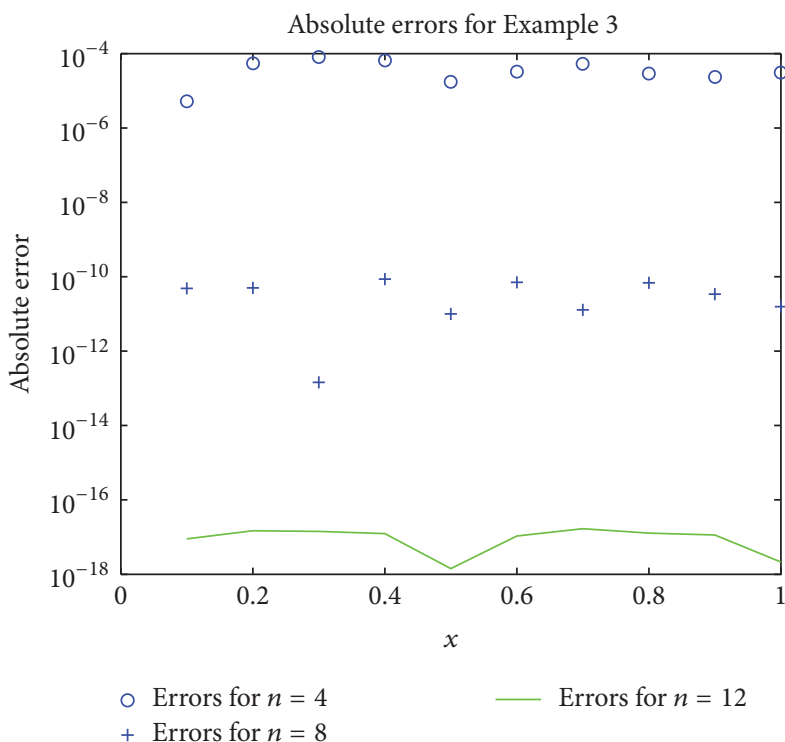

(b)

Figure 3: The approximate solution for Example 3 and its absolute errors for different $n$.

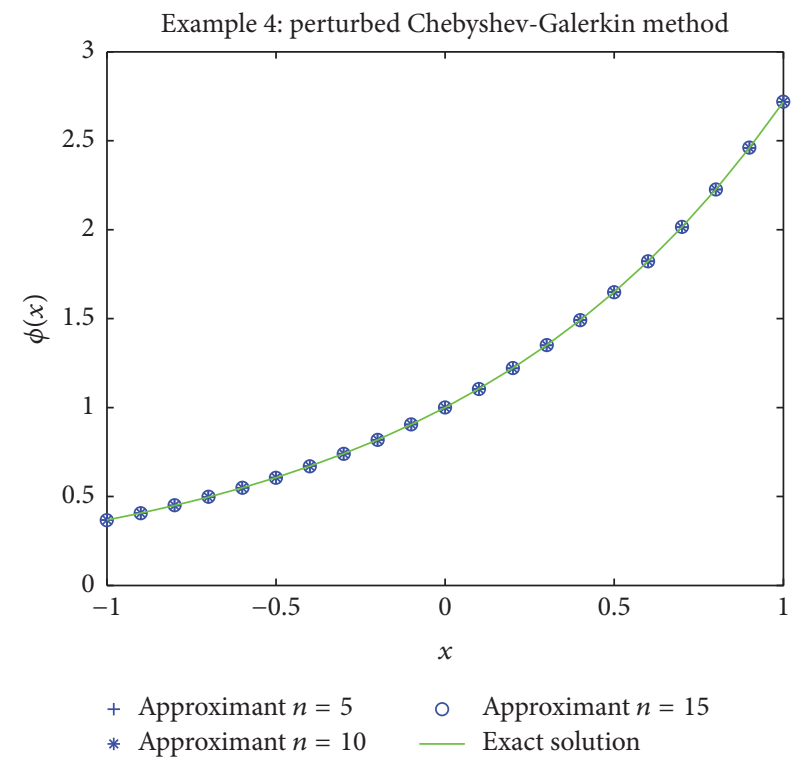

(a)

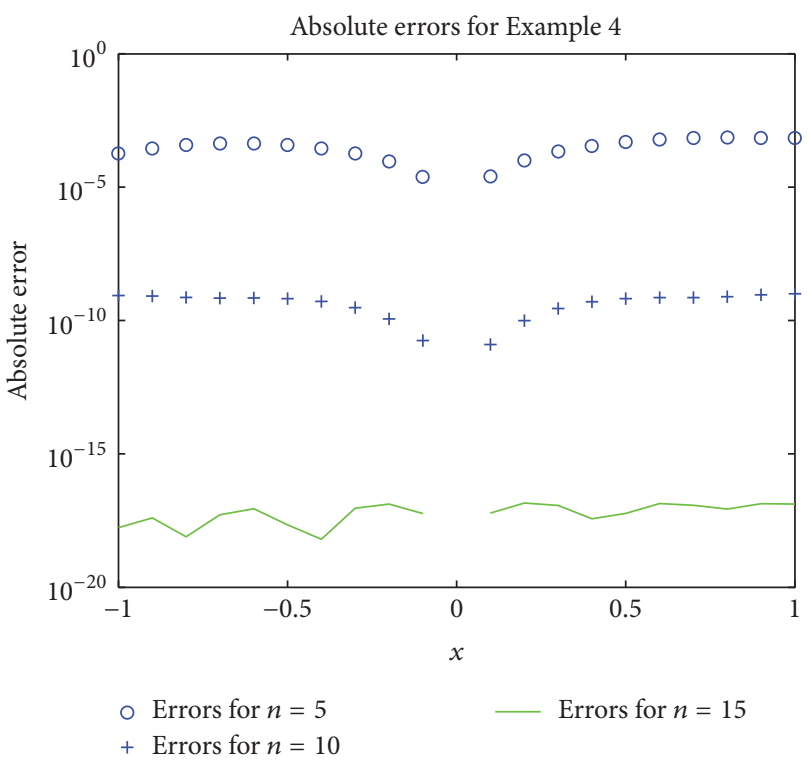

(b)

FIgURE 4: The approximate solution for Example 4 and its absolute errors for different $n$.

TABLE 6: Comparison of absolute maximum errors for Example 4 at different $n$.

\begin{tabular}{lcc}
\hline$n$ & Present method & Tau method [29] \\
\hline 5 & $7.1247 e-04$ & $3.19 e-03$ \\
10 & $1.0014 e-09$ & $2.10 e-06$ \\
15 & $1.4365 e-17$ & $7.50 e-11$ \\
\hline
\end{tabular}

\section{Competing Interests}

The authors declare that they have no competing interests.

\section{References}

[1] J. Biazar and F. Salehi, "Chebyshev Galerkin method for integrodifferential equations of the second kind," Iranian Journal of Numerical Analysis and Optimization, vol. 6, no. 1, pp. 31-42, 2016.

[2] T. Jangveladze, Z. Kiguradze, and B. Neta, "Galerkin finite element method for one nonlinear integro-differential model," Applied Mathematics and Computation, vol. 217, no. 16, pp. 6883-6892, 2011.

[3] M. Fathy, M. El-Gamel, and M. S. El-Azab, "Legendre-Galerkin method for the linear Fredholm integro-differential equations," 
Applied Mathematics and Computation, vol. 243, pp. 789-800, 2014.

[4] J. Shen, "Efficient spectral-galerkin method I. direct solvers of second- and fourth-order equations using legendre polynomials," SIAM Journal on Scientific Computing, vol. 15, no. 6, pp. 1489-1505, 1994.

[5] M. Dehghan and F. Shakeri, "Solution of an integro-differential equation arising in oscillating magnetic fields using he's homotopy perturbation method," Progress in Electromagnetics Research, vol. 78, pp. 361-376, 2008.

[6] J. Saberi-Nadjafi and A. Ghorbani, "He's homotopy perturbation method: an effective tool for solving nonlinear integral and integro-differential equations," Computers \& Mathematics with Applications, vol. 58, no. 11-12, pp. 2379-2390, 2009.

[7] S. M. Hosseini and S. Shahmorad, "Tau numerical solution of Fredholm integro-differential equations with arbitrary polynomial bases," Applied Mathematical Modelling, vol. 27, no. 2, pp. 145-154, 2003.

[8] S. M. Hosseini and S. Shahmorad, "Numerical solution of a class of integro-differential equations by the tau method with an error estimation," Applied Mathematics and Computation, vol. 136, no. 2-3, pp. 559-570, 2003.

[9] A. K. Jimoh and K. Issa, "Comparison of some numerical methods for the solution of fourth order Integro-Differential equations," Journal of the Nigerian Association of Mathematical Physics, vol. 28, pp. 115-122, 2014.

[10] N. M. Chuong and N. V. Tuan, "Spline collocation methods for FredholmVolterra integro differential equations of high order," Vietnam Journal of Mathematics, vol. 25, no. 1, p. 1524, 1997.

[11] H. Brunner, "The approximate solution of initial-value problems for general Volterra integro-differential equations," Computing. Archives for Scientific Computing, vol. 40, no. 2, pp. 125137, 1988.

[12] N. Minh and N. Van, "Spline collocation methods for Fredholm integro-differential equations of second order," Acta Mathematica Vietnamica, vol. 20, no. 1, pp. 85-98, 1995.

[13] S. Yalçinbaş and M. Sezer, “The approximate solution of highorder linear Volterra-Fredholm integro-differential equations in terms of Taylor polynomials," Applied Mathematics and Computation, vol. 112, no. 2-3, pp. 291-308, 2000.

[14] Z. Avazzadeh and M. Heydari, "Chebyshev polynomials for solving two dimensional linear and nonlinear integral equations of the second kind," Computational \& Applied Mathematics, vol. 31, no. 1, pp. 127-142, 2012.

[15] Y. Huang and X.-F. Li, "Approximate solution of a class of linear integro-differential equations by Taylor expansion method," International Journal of Computer Mathematics, vol. 87, no. 6 , pp. 1277-1288, 2010.

[16] S. Yalçinbaş and M. Sezer, "A Taylor collocation method for the approximate solution of general linear Fredholm-Volterra integro-difference equations with mixed argument," Applied Mathematics and Computation, vol. 175, no. 1, pp. 675-690, 2006.

[17] K. Maleknejad, S. Sohrabi, and Y. Rostami, "Numerical solution of nonlinear Volterra integral equations of the second kind by using Chebyshev polynomials," Applied Mathematics and Computation, vol. 188, no. 1, pp. 123-128, 2007.

[18] C. Chen and T. Shih, Finite Element Methods for IntegroDifferential Equations, World Scientific Publishing, Singapore, 1997.
[19] M. R. Crisci, E. Russo, and A. Vecchio, "Stability results for onestep discretized collocation methods in the numerical treatment of Volterra integral equations," Mathematics of Computation, vol. 58, no. 197, pp. 119-134, 1992.

[20] A. Makroglou, "Convergence of a block-by-block method for nonlinear Volterra integro-differential equations," Mathematics of Computation, vol. 35, no. 151, pp. 783-796, 1980.

[21] P. Linz, "Linear multistep methods for Volterra integrodifferential equations," Journal of the Association for Computing Machinery, vol. 16, pp. 295-301, 1969.

[22] S. H. Chang, "On certain extrapolation methods for the numerical solution of integro-differential equations," Mathematics of Computation, vol. 39, no. 159, pp. 165-171, 1982.

[23] Z. Avazzadeh, M. Heydari, and G. B. Loghmani, "Numerical solution of Fredholm integral equations of the second kind by using integral mean value theorem," Applied Mathematical Modelling, vol. 35, no. 5, pp. 2374-2383, 2011.

[24] A. H. Khater, A. B. Shamardan, D. K. Callebaut, and M. R. Sakran, "Numerical solutions of integral and integrodifferential equations using Legendre polynomials," Numerical Algorithms, vol. 46, no. 3, pp. 195-218, 2007.

[25] N. Bildik, A. Konuralp, and S. Yalçınbaş, "Comparison of Legendre polynomial approximation and variational iteration method for the solutions of general linear Fredholm integro-differential equations," Computers \& Mathematics with Applications, vol. 59, no. 6, pp. 1909-1917, 2010.

[26] A. Shahsavara, "Numerical solution of linear Volterra and Fredholm integro differential equations using Haar wavelets," Mathematical Sciences Journal, vol. 6, pp. 85-96, 2010.

[27] M. H. Fahmy, M. A. Abdou, and E. I. Deebs, "Fredholm-Volterra integral equation of the first kind with potential kernel," Le Matematiche, vol. 55, no. 1, pp. 55-74, 2000.

[28] Ş. Yüzbaşı, "Improved Bessel collocation method for linear Volterra integro-differential equations with piecewise intervals and application of a Volterra population model," Applied Mathematical Modelling, vol. 40, no. 9-10, pp. 5349-5363, 2016.

[29] S. Shahmorad, "Numerical solution of the general form linear Fredholm-Volterra integro-differential equations by the Tau method with an error estimation," Applied Mathematics and Computation, vol. 167, no. 2, pp. 1418-1429, 2005.

[30] K. Issa and R. B. Adeniyi, "Extension of generalized recursive tau method to non-linear ordinary differential equations," Journal of the Nigerian Mathematical Society, vol. 35, no. 1, pp. 18-24, 2016.

[31] P. Deulfhard, Newton Methods for Nonlinear Problems: Affine Invariant and Adaptive Algorithms, Springer, Berlin, Germany, 2004.

[32] M. H. Aliabadi and E. L. Ortiz, "A Tau method based on nonuniform space-time elements for the numerical simulation of solitons," Computers \& Mathematics with Applications, vol. 22, no. 9, pp. 7-19, 1991.

[33] A. Golbabai and S. Seifollahi, "Radial basis function networks in the numerical solution of linear integro-differential equations," Applied Mathematics and Computation, vol. 188, no. 1, pp. 427432, 2007.

[34] A. Wazwaz, Linear and Nonlinear Integral Equations, Methods and Applications, Springer, 2011. 


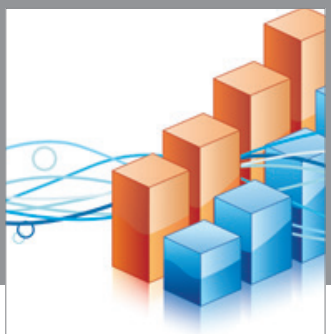

Advances in

Operations Research

vatem alat4

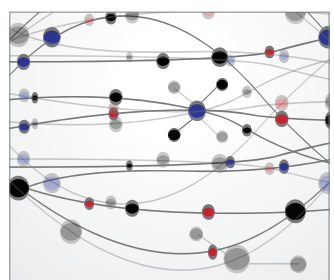

\section{The Scientific} World Journal
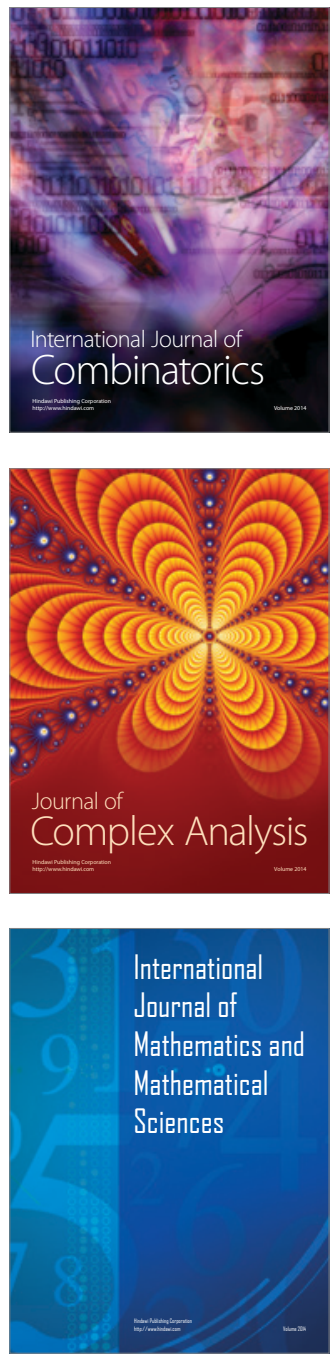
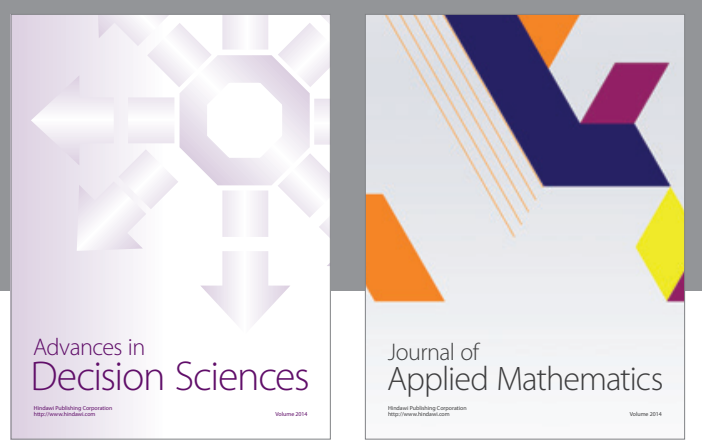

Algebra

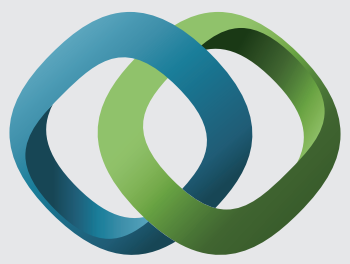

\section{Hindawi}

Submit your manuscripts at

https://www.hindawi.com
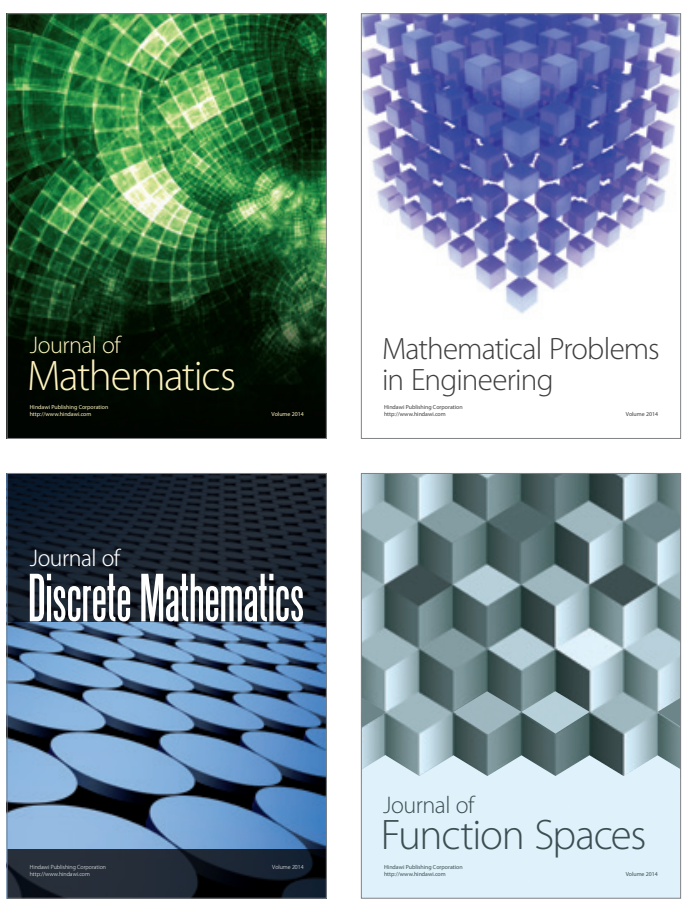

Mathematical Problems in Engineering
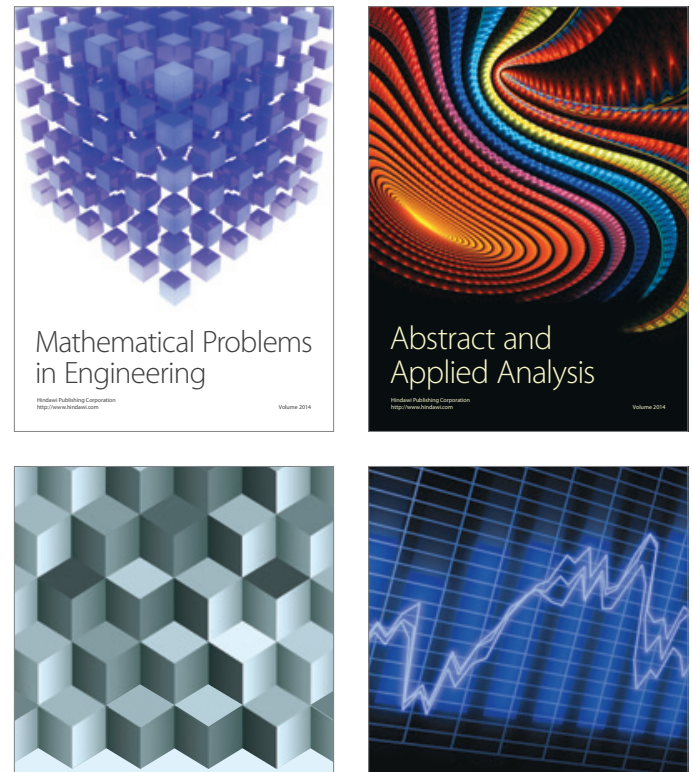

Journal of

Function Spaces

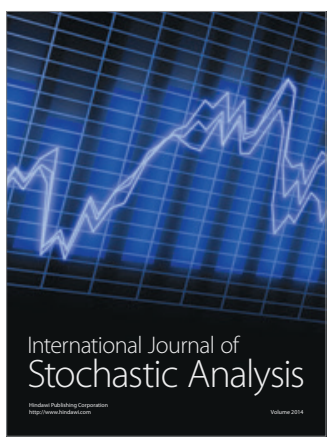

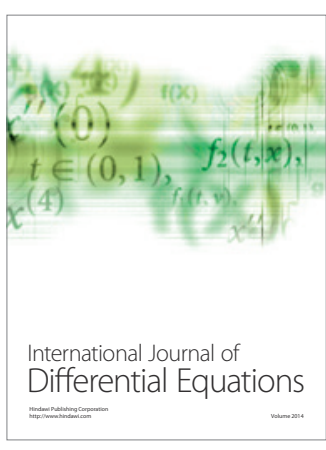
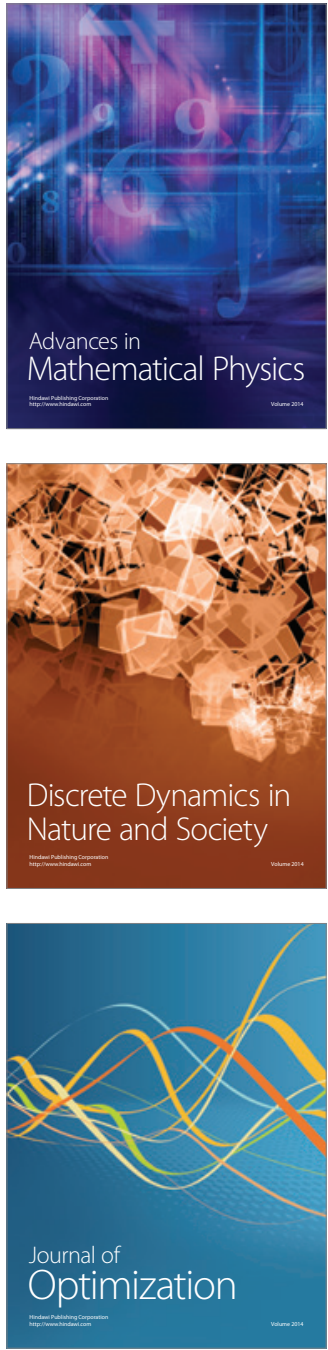